\title{
Optical Emission Spectroscopy of Electrical Discharge Machining Plasma
}

\author{
A Descoeudres ${ }^{1}$, Ch Hollenstein ${ }^{1}, \mathbf{R}$ Demellayer $^{2}$ and G \\ Wälder ${ }^{2}$ \\ ${ }^{1}$ Centre de Recherches en Physique des Plasmas, Ecole Polytechnique Fédérale de \\ Lausanne, CH-1015 Lausanne, Switzerland \\ ${ }^{2}$ Charmilles Technologies SA, 8-10 rue du Pré-de-la-Fontaine, CH-1217 Meyrin, \\ Switzerland \\ E-mail: antoine.descoeudres@epfl.ch
}

\begin{abstract}
Plasma created during Electrical Discharge Machining is systematically investigated with optical emission spectroscopy. Typical spectra show a strong $\mathrm{H}_{\alpha}$ and continuum radiation, with many lines emitted by impurities coming from electrode and workpiece materials. The dielectric molecules are cracked by the discharge. Changing polarity affects the electrode wear and workpiece erosion rates, which can be qualitatively seen on spectra. Time-resolved spectroscopy shows that plasma density reaches $2 \cdot 10^{18} \mathrm{~cm}^{-3}$ at the beginning of the discharge. This extreme density causes merging of lines, strong Stark broadening and shift of the $\mathrm{H}_{\alpha}$ line. The density decreases afterwards rapidly with time. The electron temperature remains roughly constant around $0.7 \mathrm{eV}$. The low temperature and the high density measured prove that the EDM plasma is non-ideal $(\Gamma \simeq 0.45)$. Absence of the $\mathrm{H}_{\beta}$ line, asymmetric shape of the $\mathrm{H}_{\alpha}$ line and complex structures around $\mathrm{H}_{\alpha}$ are other spectroscopic evidences of the plasma non-ideality.
\end{abstract}

\section{INTRODUCTION}

Electrical Discharge Machining (EDM) is a well known machining technique for more than fifty years. Nowadays it is widely used in a large number of industrial areas, mainly to produce molds, dies and finished parts with complex shapes.

This technology uses the eroding effect of electric sparks discharges on the electrodes $[1,2,3]$. Thus, the machining consists in successively removing small volumes of electrode material, which are molten or vaporized during the discharge. The electrical discharges are created between the conductive workpiece and a shaped electrode (diesinking machine) or a metallic wire (wire cutting machine). Thus EDM can be used to machine any material that conducts electricity, whatever its hardness may be. The sparks are created in a flowing dielectric fluid, generally water or oil, for several reasons. It guarantees a high plasma pressure and therefore a high removing force on the molten metal when the plasma collapses, it solidifies the molten metal into small particles, and it also enhances the flushing of these particles. 
Although EDM technology has been widely used for a long time, very few studies have been made on the discharge itself. During the last few years, improvements in machining speed, accuracy and roughness have been achieved with efforts on robotics, automatisation, flushing and generator design $[4,5]$. Numerous works were also done concerning modifications of the machined surface and bulk [6, 7]. But further EDM improvements, especially for micro-machining, require a better control of the discharge and of its interaction with electrodes. It is essential to understand the basic physics of the spark immersed in a dielectric, which is the heart of the process.

The physical phenomena involved in EDM process are complex. Few theoretical and numerical studies have been made [8, 9, 10, 11], and experimental characterization of the plasma is lacking. Optical Emission Spectroscopy is a straightforward diagnostic to obtain physical information about the discharge, such as plasma composition, temperatures, density or pressure. However, experimental difficulties arise due to the low emission intensity, the short duration of the discharges, the small size of the plasma and the effect of the dielectric. The low light intensity makes measurement on a single discharge difficult. The light detection has to be done over numerous discharges, but the sparks are often not reproducible for same experimental conditions, due to the constant evolution of the dielectric cleanliness and of the electrodes surface and geometry. A spatial characterization of the plasma demands a certain miniaturization of the light acquisition tools. Other intrusive diagnostics are not applicable, also due to the small size of the plasma. Some spectroscopic measurements have already been made but remain very incomplete $[12,13]$.

In various fields, breakdown in dielectric liquids or solid have already been studied: pre-breakdown and breakdown in different liquids [14, 15] and condensed matter [16], exploding wires in water [17], laser produced plasma in transformer oil [18], and Teflon capillary discharges [19] for example. These plasmas are similar to the EDM plasma, and their typical density and temperature show that they are non-ideal or strongly coupled plasmas. Such plasmas have very interesting physical properties and remain not well known [20, 21].

In this paper we present a systematic investigation of the effects of the plasma parameters on optical emission spectra. First, time-integrated spectra are shown for different dielectrics, electrode materials, polarity and on-time. We also present timeresolved spectra and deduce from them the evolution of plasma density and temperature. Finally, along with density and temperature measurements and several spectroscopic observations, we show that the EDM plasma is a non-ideal plasma.

\section{EXPERIMENTAL SETUP}

Figure 1 shows a schematic drawing of the experimental setup. We use a small and versatile die-sinking EDM machine, with 3 or $5 \mathrm{~mm}$ diameter cylindrical electrodes. In order to better control the localization of the sparks, the electrode tip is conical and the servo-controlled movement of the electrode is purely vertical. The dielectric, the 
electrode and the workpiece can easily be changed. We use pure water, mineral oil and liquid nitrogen as dielectric; copper, tungsten and graphite electrodes; and W300 steel workpieces. When machining with liquid nitrogen, the workpiece is placed in a dewar to avoid boiling as much as possible. All the discharge parameters can be controlled with the generator: voltage, current, on-time, off-time, polarity and movements of the electrode. The distance between the electrode and the workpiece during a discharge is an important parameter, but it is difficult to measure it. The order of magnitude can be estimated around 10 to $100 \mu \mathrm{m}$.

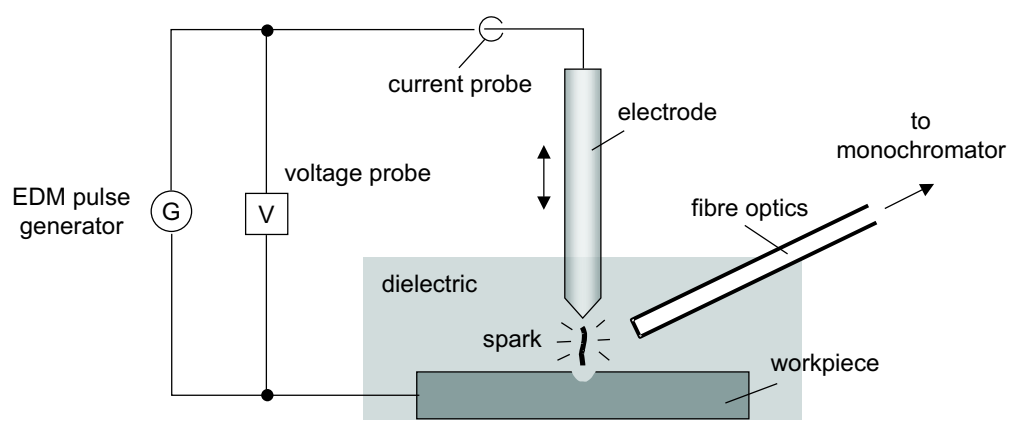

Figure 1. Schematic drawing of the experimental setup

Figure 2 is a close-up image of the electrodes during machining. This image was obtained with an endoscope composed of 10'000 fibres coupled to a CCD camera. The plasma is clearly visible, along with gas bubbles created by the discharge.

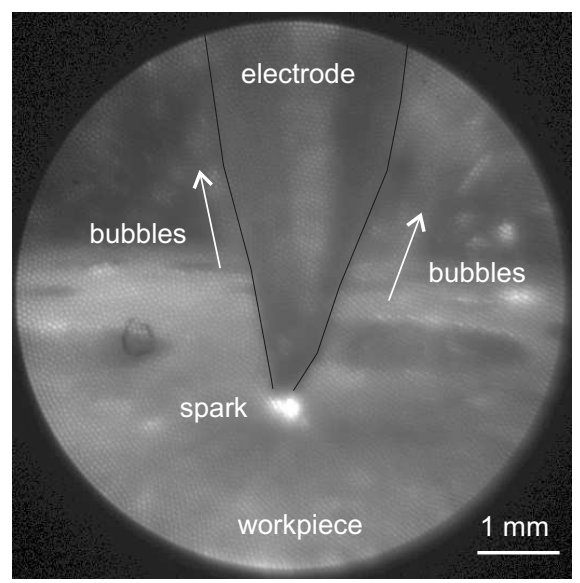

Figure 2. Image of a discharge in water obtained with an endoscope (copper electrode, steel workpiece, discharge current $6 \mathrm{~A}$, discharge on-time $6.4 \mu \mathrm{s}$ )

Current and voltage characteristics of the discharge are measured with a high frequency current probe and a differential voltage probe connected to a fast oscilloscope. For Optical Emission Spectroscopy, the emitted light is collected by a quartz fibre. The fibre is immersed in the dielectric, located a few millimeters from the spark to optimize 
the collected light intensity and to reduce absorption from the dielectric. The light is dispersed by a $0.275 \mathrm{~m}$ monochromator equipped with three gratings (150, 600 and 1200 $\mathrm{g} / \mathrm{mm}$ ). The resolving power of the spectrograph (apparatus function) is respectively $1.25,0.35$ and $0.17 \mathrm{~nm}$. For time-integrated spectra, the detector was a CCD camera (1024 x 256 pixels); for time-resolved spectra, the detector was an array of 700 gated photodiodes. For both detectors, the resolution per pixel is about 0.6, 0.15 and $0.06 \mathrm{~nm}$ respectively. The spectral range is approximately 300 to $1000 \mathrm{~nm}$. In addition, a fast photomultiplier is also used to measure the time evolution of the total light intensity. The spectral sensitivity of the optical system (fibre + monochromator + CCD camera) has been measured with a reference light source for different conditions: without dielectric, with clean oil and with oil contaminated by eroded particles. Though the light absorption by the dielectric increases drastically in contaminated oil, the relative spectral sensitivity remains fairly constant without oil, in clean or contaminated oil. The spectral sensitivity is almost constant between 500 and $900 \mathrm{~nm}$, but we have a loss of sensitivity under $500 \mathrm{~nm}$ and above $900 \mathrm{~nm}$ mainly due to the quantum efficiency of the CCD camera.

\section{RESULTS AND DISCUSSION}

\subsection{Time-integrated emission spectra}

In this section, we present time-integrated emission spectra. The light of thousands sparks is accumulated over several seconds.

A typical spectrum from EDM discharge is shown in figure 3. The experimental conditions such as electrode material/workpiece material, dielectric, discharge current and discharge on-time are given in the figure captions. The dominant line is the Balmer $\mathrm{H}_{\alpha}$ line emitted by atomic hydrogen, which comes from the cracking of the dielectric molecules. Some lines of atomic carbon and $\mathrm{C}_{2}$ molecules (Swan Bands) are also visible. This indicates that the organic molecules of the dielectric are almost completely cracked by the discharge. The plasma is contaminated by impurities: several lines of atomic copper from the electrode are present, along with many lines originating from atomic iron, chromium and carbon of the removed material of the steel workpiece. The fact that all observed lines are atomic lines (and a few neutral molecular lines) and that no ionic lines are visible is a first indication that the plasma is cold. If the plasma was not cold, ionic lines of iron or copper would be present, because the atoms of metals are easily ionized. The strong continuum radiation is due to free-bound transitions. Although the $\mathrm{H}_{\alpha}$ line is dominant, the remaining Balmer series lines such as $\mathrm{H}_{\beta}$ or $\mathrm{H}_{\gamma}$ are not observed. Furthermore, the $\mathrm{H}_{\alpha}$ line is broadened. These remarkable points will be discussed later.

The effect of the dielectric on emission spectra is shown in figure 4. As mentioned previously, the discharge cracks the dielectric molecules, even the strong triple bond of nitrogen. We observe atomic hydrogen and oxygen lines from water; atomic hydrogen, 


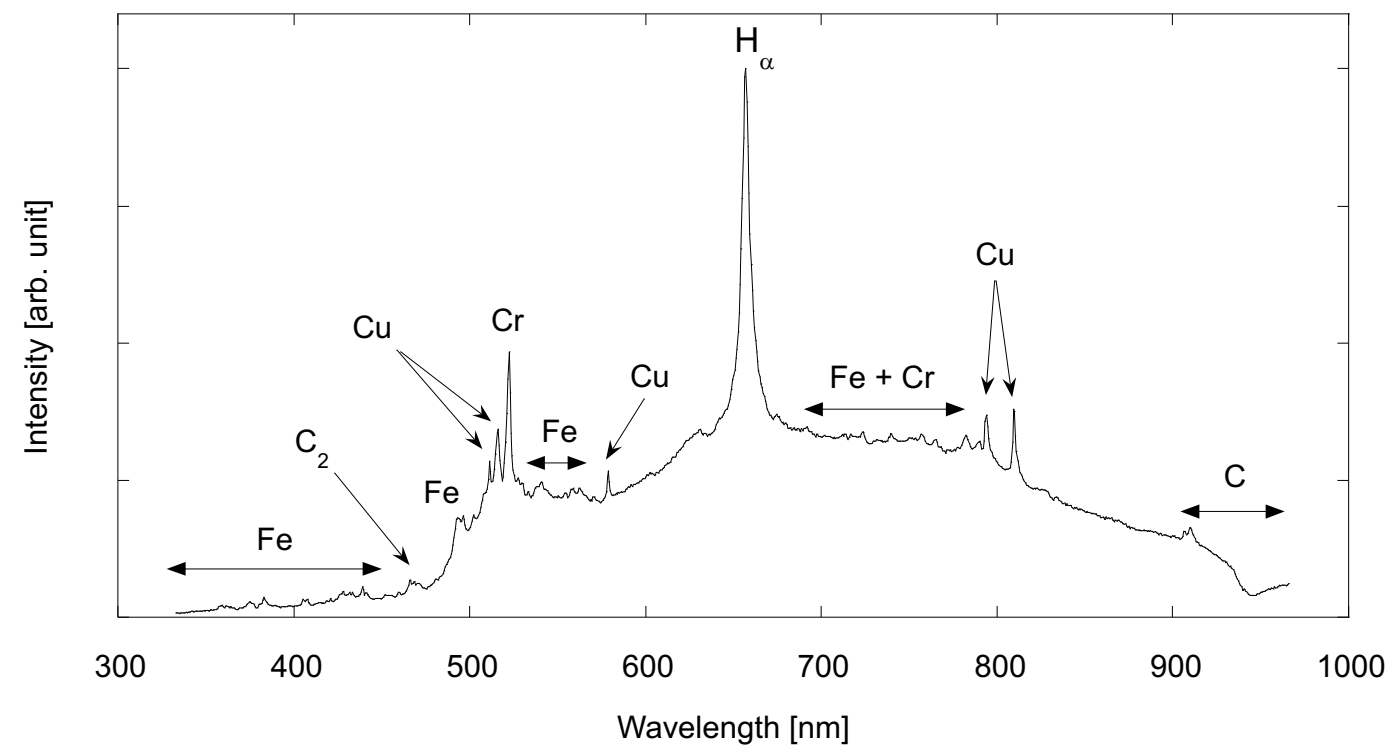

Figure 3. Typical emission spectrum $(\mathrm{Cu} / \mathrm{steel}$, oil, $12 \mathrm{~A}, 2 \mu \mathrm{s}, 150 \mathrm{~g} / \mathrm{mm}$ grating $)$

atomic carbon and $\mathrm{C}_{2}$ lines from oil; atomic nitrogen lines from liquid nitrogen. The dominant lines in figure 4 (c) are copper lines, because we have more electrode wear than workpiece erosion under these experimental conditions in liquid nitrogen.
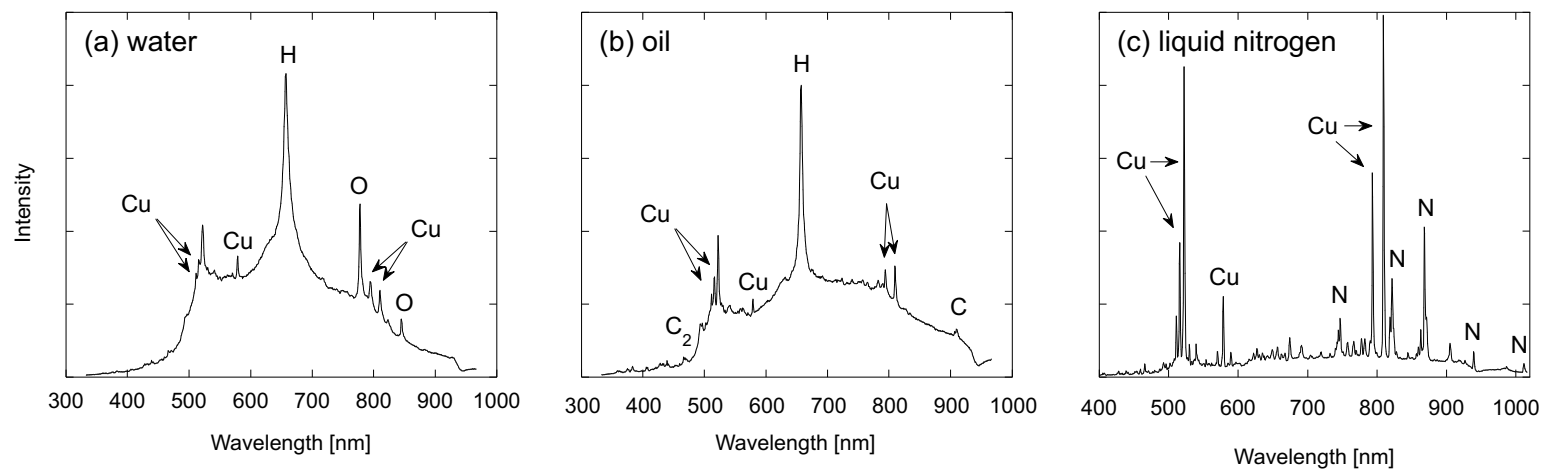

Figure 4. Effect of the dielectric on the emission spectrum $(\mathrm{Cu} / \mathrm{steel}, 150 \mathrm{~g} / \mathrm{mm}$ grating, (a) water, $12 \mathrm{~A}, 2 \mu \mathrm{s}$; (b) oil, $12 \mathrm{~A}, 2 \mu \mathrm{s}$; (c) liquid nitrogen, $48 \mathrm{~A}, 2 \mu \mathrm{s}$ )

The effect of electrode material on emission spectra is shown in figure 5. The electrode material contaminates the plasma, and lines of respectively atomic copper, of atomic carbon and $\mathrm{C}_{2}$, and of atomic tungsten are emitted. With a graphite electrode, the Swan bands of $\mathrm{C}_{2}$ and the $\mathrm{C}$ lines are much stronger than in figures 3 and 4 , because the carbon contamination of the plasma due to the graphite electrode is much greater than the carbon produced by the cracking of the oil. The tungsten lines are very difficult to observe because they are located in the same spectral region as many iron lines, which are more intense.

The emission spectra give also qualitative information about the electrode wear or the 

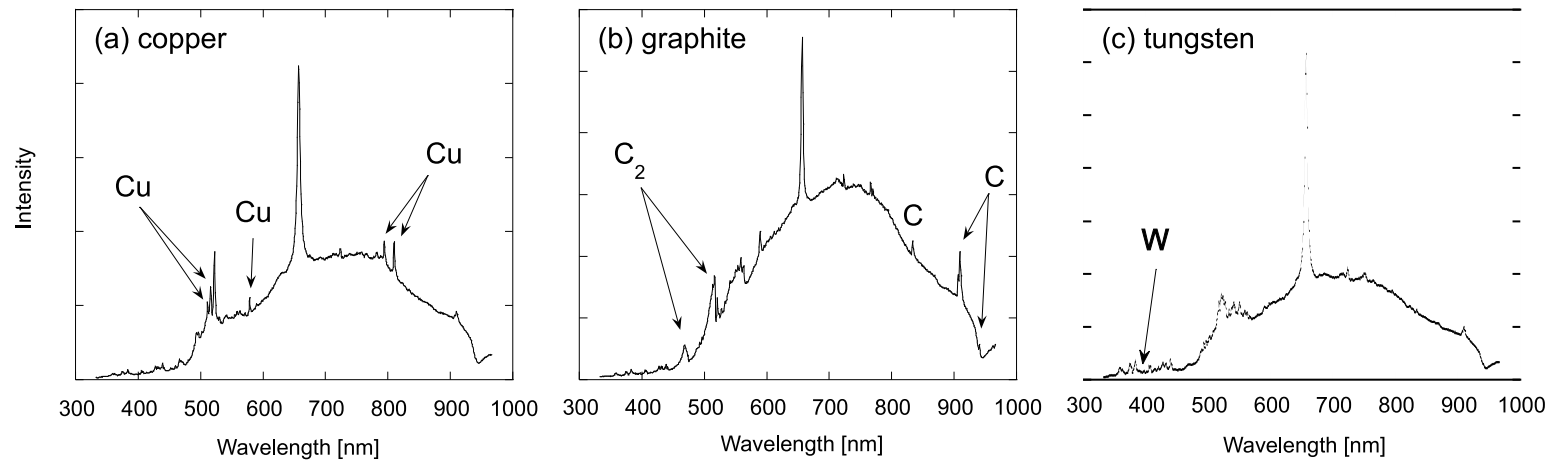

Figure 5. Effect of the electrode material on the emission spectrum (oil, 12A, $5 \mu \mathrm{s}$, $150 \mathrm{~g} / \mathrm{mm}$ grating, (a) Cu/steel; (b) graphite/steel; (c) W/steel)

workpiece erosion. Figure 6 shows two spectra measured under the same experimental conditions, except for the electrode polarity. In figure 6 (a), the tungsten electrode is positively polarized with respect to the workpiece. In this configuration, the wear of the electrode is very strong. In the other configuration where the workpiece is positively polarized with respect to the electrode (figure 6 (b)), the erosion is more effective and the electrode wears much less. The polarity has a clear influence on spectra. With negative electrode polarity, the iron lines of the group around $520 \mathrm{~nm}$ are much more intense than with positive polarity. If we consider the $\mathrm{H}_{\alpha}$ line as a reference of intensity (its intensity does not depend on erosion or wear but originates only from the dielectric), the iron lines show clearly that the erosion is more efficient with negative polarity. If we look at the region between 350 and $450 \mathrm{~nm}$, it is obvious that the wear is stronger with positive polarity: in (a) several tungsten and iron lines are visible with comparable intensities, in (b) the tungsten lines are no more visible and the iron lines are more intense. Note that these conclusions are only qualitative, since it is difficult to deduce from these spectra the quantities of materials removed.
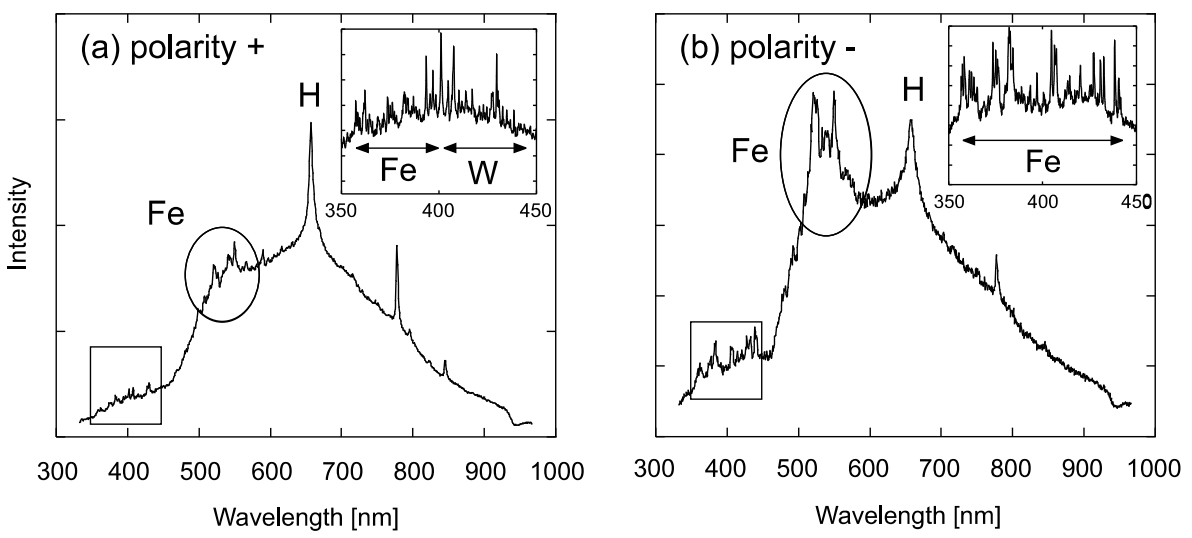

Figure 6. Effect of the electrode polarity on the emission spectrum (W/steel, water, $1 \mathrm{~A}, 100 \mu \mathrm{s}, 150$ and $600 \mathrm{~g} / \mathrm{mm}$ gratings) 
The discharge on-time has also a significant influence on emission spectra. Figure 7 (a) and (b) are spectra for two different on-times of discharges where the current is controlled and maintained constant during the discharge. To produce shorter discharges, we use "capacitive discharges": a capacitor is mounted in parallel with the electrode-workpiece gap, and its discharge creates very short sparks. In this case, the current and the ontime are not controlled. Figure 7 (c) is the spectrum of capacitive discharges produced with a $10 \mathrm{nF}$ capacitor. The duration of plasma created with these capacitive discharges is approximately $1.6 \mu \mathrm{s}$ and the current reaches approximately $6 \mathrm{~A}$.
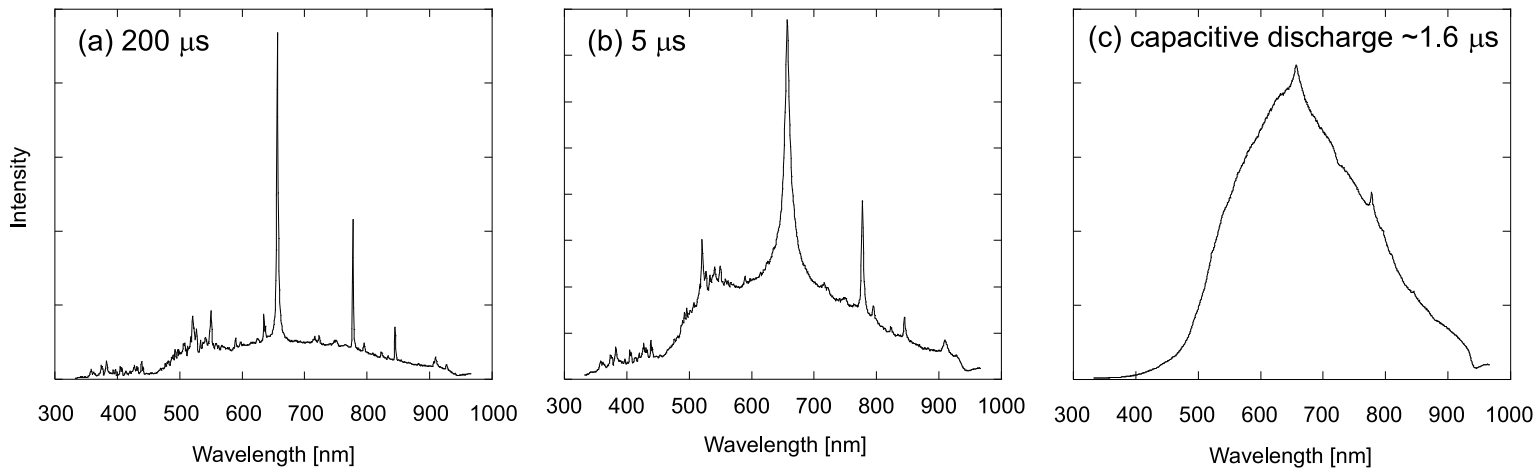

Figure 7. Effect of the on-time on the emission spectrum $(\mathrm{Cu} / \mathrm{steel}$, water, $150 \mathrm{~g} / \mathrm{mm}$ grating, (a) $12 \mathrm{~A}, 200 \mu \mathrm{s}$; (b) $12 \mathrm{~A}, 5 \mu \mathrm{s}$; (c) capacitive discharge, $\sim 1.6 \mu \mathrm{s}$ )

The broadening of the $\mathrm{H}_{\alpha}$ line increases drastically with decreasing on-time as shown in figure 7 (a) and (b). The Full Width at Half Maximum (FWHM) of the $\mathrm{H}_{\alpha}$ line reaches almost $10 \mathrm{~nm}$ in figure 7 (b). Such an important broadening can only be due to dynamic Stark broadening, which dominates by far pressure broadening or Doppler broadening in these conditions $[18,22]$. The hydrogen line is more strongly broadened than other atomic lines, because the hydrogen atom is more sensitive to the Stark effect than other atoms. Stark broadening of the $\mathrm{H}_{\alpha}$ line depends strongly on the electron density and can be used to evaluate it [23]. The $\mathrm{H}_{\alpha}$ line is also shifted to higher wavelengths with increasing electron density. The measured values of FWHM and line shift are a sign of an extremely high electron density, especially for brief discharges and thus for the beginning of any discharge. Because the presented spectra are time-integrated, the evolution of electron density can not be directly determined from the FWHM measurements.

For discharges shorter than $2 \mu \mathrm{s}$, the plasma is so dense that almost all the atomic lines are completely merged into one broad continuum. We hardly distinguish on figure 7 (c) the $\mathrm{H}_{\alpha}$ line, two oxygen lines and two copper lines. Such extreme densities destroy the upper energy levels of the atoms, and the free-bound radiation becomes more important than line radiation [24, 25]. As discussed in paragraph 3.3., this is spectroscopic evidence that the plasma created during the earliest time of an EDM discharge is a non-ideal plasma. 


\subsection{Time-resolved emission spectra}

Time-resolved spectra are obtained with gated photodiodes. The light is detected only during a short period of the discharge, which is the time resolution of the measurement. Changing the delay between the beginning of the discharge and the beginning of the light acquisition, we obtain a succession of spectra. Each spectrum is obtained accumulating the light of the same part of thousands sparks.

Time-resolved measurements confirm the previous conclusions about the high plasma density. Figure 8 shows time-resolved emission spectra of a spectral region where numerous metal lines are visible. The spectra are normalized in intensity. The iron, chromium and copper lines are completely merged during approximately the first microsecond, which indicates again a particularly high electron density.

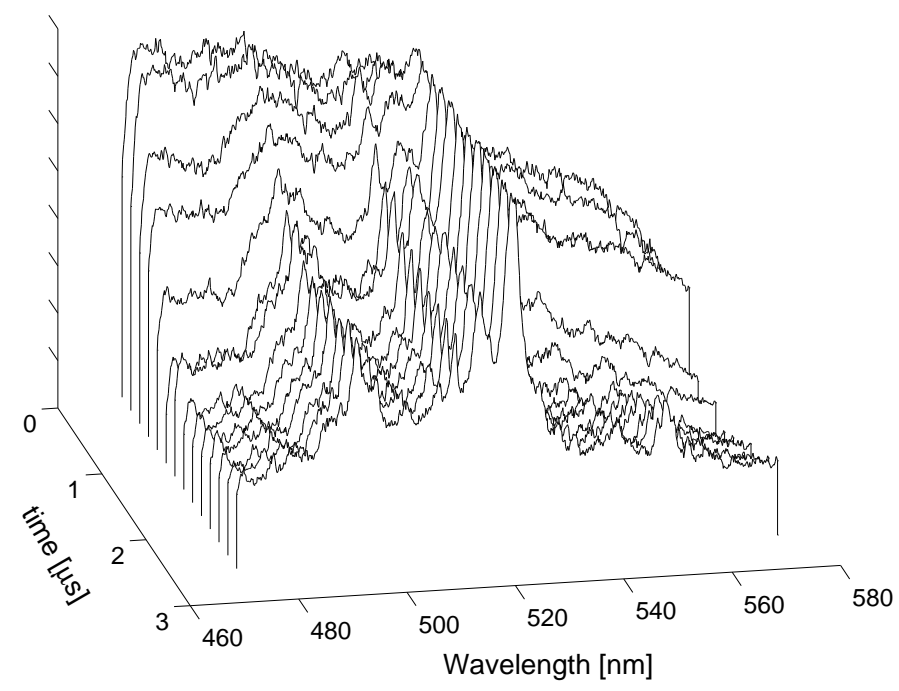

Figure 8. Merging of the $\mathrm{Fe}, \mathrm{Cu}$ and $\mathrm{Cr}$ atomic lines $(\mathrm{Cu} /$ steel, water, $12 \mathrm{~A}$, time resolution $200 \mathrm{~ns}, 600 \mathrm{~g} / \mathrm{mm}$ grating)

Three copper lines within this spectral region and of which line parameters are given in table 1 have been used for electron temperature calculation with the Boltzmann plot method. This method requires a pair of lines emitted by the same species with a large difference in excited state energy. Assuming local thermal equilibrium (LTE), the temperature $T$ is then given by

$$
T=\frac{E_{2}-E_{1}}{k_{B}} \cdot\left[\ln \left(\frac{I_{1} \lambda_{1} g_{2} A_{2}}{I_{2} \lambda_{2} g_{1} A_{1}}\right)\right]^{-1}
$$

where $k_{B}$ is the Boltzmann constant, $I_{i}$ the measured intensity of the line $i, \lambda_{i}$ its wavelength, $E_{i}$ the energy of the excited state, $g_{i}$ its statistical weight and $A_{i}$ the transition probability [23]. Here we can use the intensity ratio measurements of lines (a) and (b), and of lines (a) and (c). Since these lines are located in the same spectral region, we can assume that the overall spectral transmission is the same for the three 
lines.

It should be noted that the condition of LTE is not an obvious assumption, especially for a non-ideal plasma. As discussed in paragraph 3.3., thermal interactions are comparable to coulombian interactions in such plasmas. The evolution to the equilibrium state by thermal interactions is thus perturbed by coulombian effects. But in order to get a first electron temperature estimation, the assumption of LTE can reasonably be accepted.

Table 1. Copper line parameters for temperature determination.

\begin{tabular}{ccccc}
\hline Line & $\lambda[\mathrm{nm}]$ & $E[\mathrm{eV}]$ & $g$ & $A\left[10^{-8} \mathrm{~s}^{-1}\right]$ \\
\hline (a) & 510.554 & 3.82 & 4 & 0.02 \\
(b) & 515.324 & 6.19 & 4 & 0.6 \\
(c) & 521.820 & 6.19 & 6 & 0.75 \\
\hline
\end{tabular}

The electron temperature found is about $0.7 \pm 0.15 \mathrm{eV}(\sim 8100 \pm 1750 \mathrm{~K})$ and remains rather constant within the margin of error during the discharge even after $1 \mathrm{~ms}$. The errors in intensity measurements are principally due to other overlapping lines located near the copper lines (a line deconvolution must be performed). It is also difficult to measure $T$ in the beginning of the discharge, because of the line merging. Moreover, the LTE is certainly not reached during the early stage of the discharge and the equation (1) cannot be used. The temperature value of $0.7 \mathrm{eV}$ is a low electron temperature, but consistent with previous studies on EDM plasmas $[11,12]$ and other similar plasmas $[15,18,26]$. The plasma is very dense, and thus electrons rapidly lose energy by numerous collisions. This leads to a low electron temperature. Because no ionic lines are visible, it is also impossible to calculate the ion temperature. But again, due to the high plasma density, one can assume that the numerous collisions between particles thermalize them rapidly. So the ion temperature should be of the same order as the electron temperature. It should be noted that this temperature estimation does not give information about the temperature at the electrodes, which would be a very useful value to know for simulations and practical applications.

To calculate the evolution of the electron density, we use FWHM and shift measurements from time-resolved spectra of the $\mathrm{H}_{\alpha}$ line, shown in figure 9. The FWHM and shift are calculated from a lorentzian fit of the $\mathrm{H}_{\alpha}$ peak for each spectrum. The very first spectra show extremely broadened and shifted $\mathrm{H}_{\alpha}$ lines. Thus it is difficult to fit well the first spectra. The black lines on figure 9 represent the theoretical position of the $\mathrm{H}_{\alpha}$ line $(656.28 \mathrm{~nm})$ and the measured peak positions. These lines are drawn to easily visualize the line shift. At the beginning of the discharge, we can measure FWHM up to $10 \mathrm{~nm}$ and shift up to $10 \AA$.

Numerous theoretical and experimental works have been published about Stark broadening and shift of the $\mathrm{H}_{\alpha}$ line, especially for electron density calculations from FWHM [27, 28, 29, 30] and shift measurements [28, 29, 30, 31, 32]. This type of diagnostic is particularly important in astrophysics. Computation of spectral lineshapes 


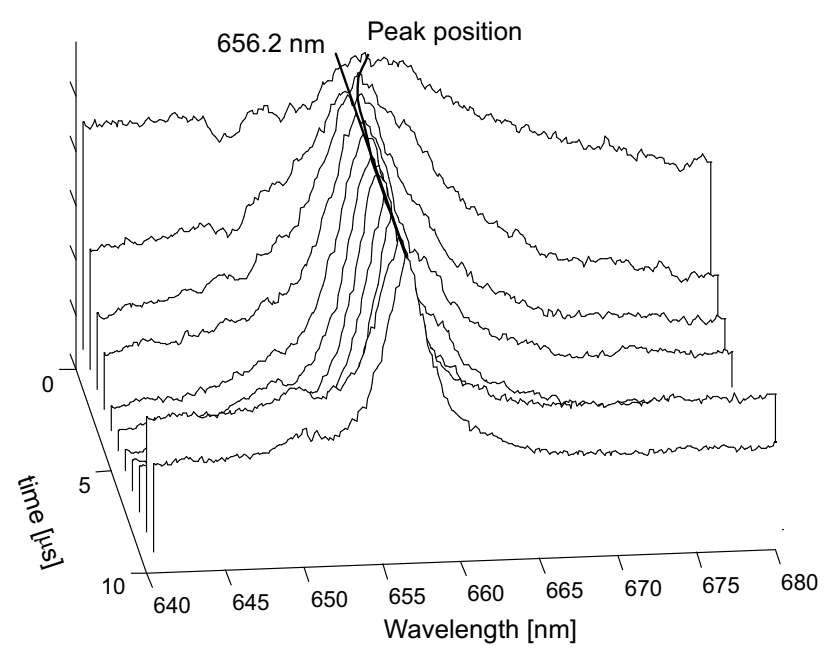

Figure 9. Broadening and shift of the $\mathrm{H}_{\alpha}$ line $(\mathrm{Cu} /$ steel, water, $16 \mathrm{~A}$, time resolution $1 \mu \mathrm{s}, 600 \mathrm{~g} / \mathrm{mm}$ grating)

is quite complex, because collisions of both electrons and ions with the emitter have to be taken into account as the broadening mechanism. But in recent years progress has been made to include several dynamic effects in calculations, especially ion dynamics. Here we used the theories of Gigosos and Cardenoso on broadening [27] and of Oks on shift [30] to calculate the electron density evolution as shown in figure 10. In spite of some differences, the FWHM and the shift measurements give comparable density values and evolution. As expected, the electron density is extremely high during the first microsecond (above $10^{18} \mathrm{~cm}^{-3}$ ) and then decreases rapidly with time. In the very beginning of the discharge, the plasma has to overcome the extreme pressure imposed by the dielectric. This leads to such a high plasma density. Assuming a temperature of $0.7 \mathrm{eV}$, a density of $2 \cdot 10^{18} \mathrm{~cm}^{-3}$ and that the plasma is a fully ionized perfect hydrogen gas, we can estimate that the plasma pressure is around 4.5 bar at the beginning of the discharge. If then the plasma column expands, density and pressure decrease. Like temperature measurements, these density measurements are consistent with EDM simulation [11] and works on similar plasmas [15, 18, 26]. On the other hand, the plasma pressure found is lower than the pressure calculated with the simulation [11]. But the assumption of a fully ionized perfect hydrogen gas is obviously a simplification. In reality the plasma composition is more complex and the plasma is partially ionized (the $\mathrm{H}_{\alpha}$ line is emitted by neutral hydrogen atoms). The pressure is thus higher than 4.5 bar, depending on the ionization degree of the plasma. Nevertheless, the pressure is still much lower than the value found with the simulation.

Intensities of spectra in figure 8 and 9 are normalized to their maximum. A photomultiplier without monochromator is used to measure directly the evolution of the light intensity. Figure 11 shows the evolution of the light intensity along with the evolution of the current and of the voltage. These three measurements are made during 


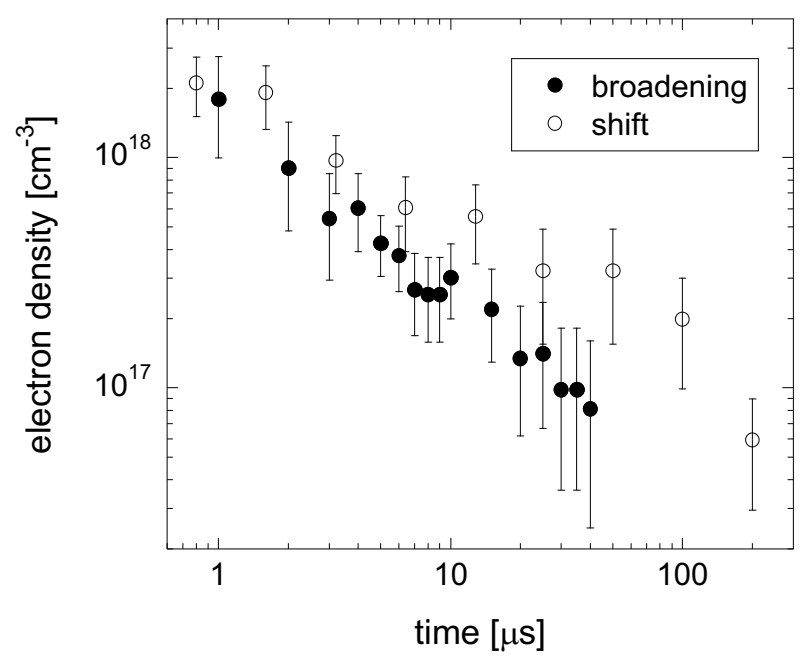

Figure 10. Electron density deduced from broadening and shift measurements of the $\mathrm{H}_{\alpha}$ line

a single discharge. The oscillations on current and voltage measurements are caused by the current regulation of the generator. Generally, the oscillations observed on current, voltage and light intensity measurements are always clearly correlated. This shows that these three physical values are unsurprisingly interdependent. Two phases can be distinguished in figure 11: the breakdown phase and the discharge.

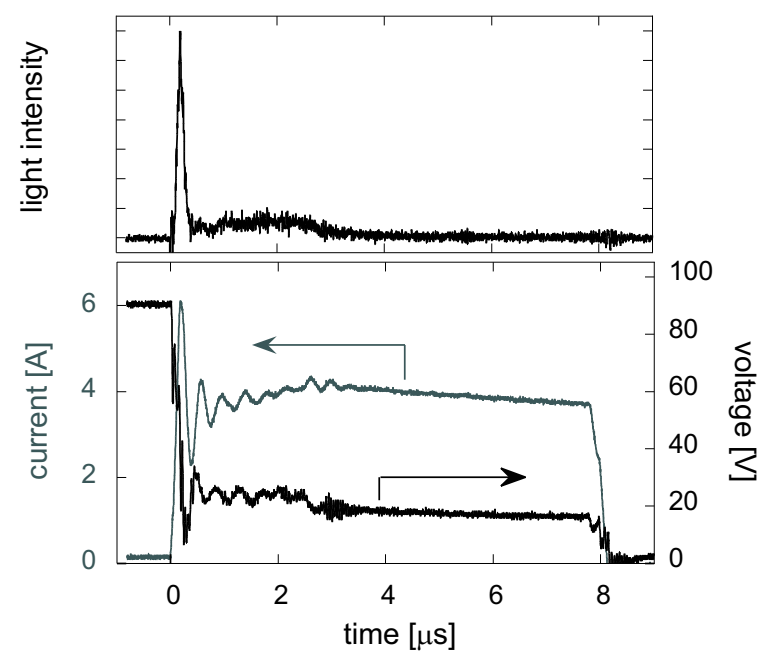

Figure 11. Evolution of the total light intensity, of the current and of the voltage during a single discharge $(\mathrm{Cu} /$ steel, water, $4 \mathrm{~A}, 8 \mu \mathrm{s})$

The major part of the light is emitted during the breakdown and approximately during the first 500 ns. Studies of pre-breakdown phenomena in dielectric liquids show that different types of streamer exist [14, 15, 33, 34]. Their structures, velocities and current depend on many parameters such as the discharge voltage and current, the electrode shape and polarity, the gap distance, the chemical and physical properties of 
the dielectric and its contamination, for example. Before the breakdown, the streamer grows rapidly and emits a weak light. If the streamer reaches the other electrode, the light emitted is very intense. The shape of the light emission is a peak as observed in figure 11. During this first phase of the discharge, the streamer brings a massive flow of energy to the workpiece. Due to the short scale of time of the streamer processes, it is probable that only the workpiece surface is affected by this primary energy flow while the workpiece bulk remains unaffected. During the discharge itself (after $500 \mathrm{~ns}$ ), the light emission is 10 to 150 times less intense. The workpiece bulk can then be affected by the plasma heating.

\subsection{Non-ideality of the plasma}

Knowing the density $n$ and the temperature $T$, we can calculate the plasma coupling parameter $\Gamma$ defined by

$$
\Gamma=\frac{Z^{2} e^{2}}{4 \pi \varepsilon_{0} a k_{B} T}
$$

where $Z$ is the charge of the ions ( $Z=1$ for hydrogen ions), $e$ the elementary charge, $\varepsilon_{0}$ the vacuum permittivity and $a$ the mean interparticle distance given by

$$
a=\left(\frac{3}{4 \pi n}\right)^{1 / 3}
$$

The plasma parameter represents the ratio of Coulomb interaction divided by thermal interaction. If $\Gamma \ll 1$, the plasma is ideal; if $\Gamma \leq 1$, the plasma is weakly non-ideal; if $\Gamma>1$, the plasma is strongly coupled. Since

$$
\Gamma \propto \frac{n^{1 / 3}}{T}
$$

non-ideal and strongly coupled plasmas are dense and cold plasmas. In such plasmas, the interparticle distance is short enough and the particle velocity is small enough to produce important electrostatic interactions between the charged particles. From our previous density and temperature measurements, the plasma parameter $\Gamma$ is around 0.45. Thus, EDM discharges produce cold and dense plasmas, which are weakly nonideal. In addition to calculation of $\Gamma$, spectroscopic results indicate directly that the plasma is non-ideal.

First of all, the extreme density lowers the ionization energy threshold and thus "erases" the upper energy levels of atoms. This results in absence of the $\mathrm{H}_{\beta}$ and $\mathrm{H}_{\gamma}$ lines for example, along with an increase in continuum radiation [20, 25]. These effects can only occur in non-ideal plasma and can clearly be observed on our spectra as said previously. Furthermore, the Inglis-Teller relation gives a rough estimation of the density from the last hydrogen Balmer line discernible:

$$
\log n=23.26-7.5 \cdot \log n_{\max }
$$

where $n$ is the density expressed in $\mathrm{cm}^{-3}$ and $n_{\max }$ the quantum number of the upper energy level of the last visible transition $[20,35,36,37]$. This relation is mainly used in 
astrophysics, to evaluate the electron density in the atmosphere of stars, for example. If we observe on EDM spectra the $\mathrm{H}_{\alpha}$ line (transition from level 3 to 2 ) but no $\mathrm{H}_{\beta}$ line (transition from level 4 to 2 ), then

$$
3 \leq n_{\max }<4
$$

and the plasma density is at least $6 \cdot 10^{18} \mathrm{~cm}^{-3}$ according to this relation. This estimation is of the same order of magnitude as our previous density measurements and confirms their validity.

In addition to broadening and shift, the $\mathrm{H}_{\alpha}$ line gives other useful information. Figure 12 shows a typical example of a spectrum around the $\mathrm{H}_{\alpha}$ line. If a lorentzian fit is applied, it is observed that the shape of the line is slightly asymmetric and deviates from the fit. This asymmetry is due to interactions between ions and the emitter (hydrogen atom in our case), and is another sign of plasma non-ideality [20, 29, 31].

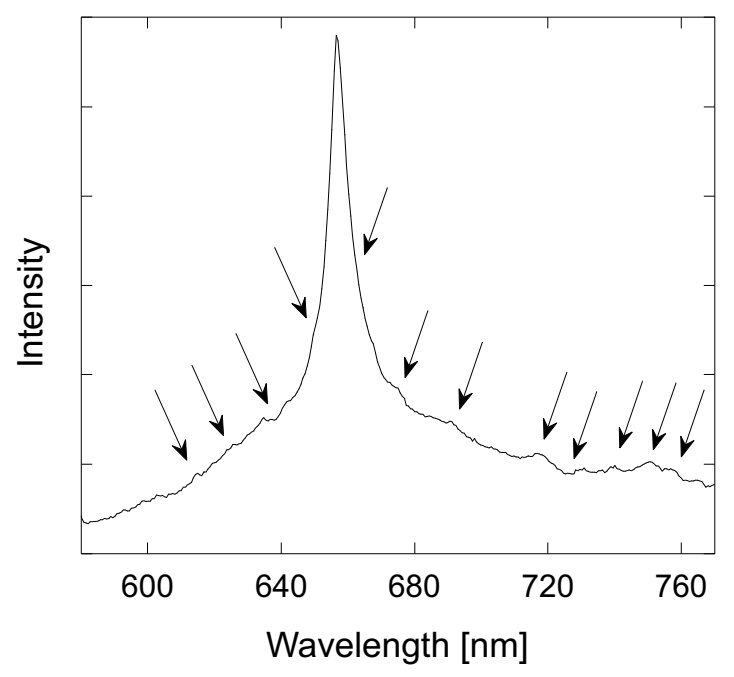

Figure 12. Complex structures around the $\mathrm{H}_{\alpha}$ line $(\mathrm{Cu} /$ steel, water, $12 \mathrm{~A}, 2 \mu \mathrm{s}$, $150 \mathrm{~g} / \mathrm{mm}$ grating)

We also see complex structures around the line. The arrows on figure 12 mark small bumps and depressions, which are not due to line radiation or absorption. These structures are created by several phenomena, which occur in non-ideal plasma. First, the electric field in the plasma causes splitting of energy levels in hydrogen atoms (Stark effect) [38]. Transitions between these new levels create several lines around $\mathrm{H}_{\alpha}$. Furthermore, if Langmuir or ion acoustic turbulence is present in the plasma, an oscillating electric field is superimposed on the static field. In the case of Langmuir turbulence, the turbulence creates satellite lines located at $656.2 \mathrm{~nm} \pm n \cdot \lambda_{p}$, where $n=1,2, \ldots$ and $\lambda_{p}$ is the wavelength associated with the Langmuir wave frequency ( $\sim 10 \mathrm{~nm}$ under our conditions). Finally, if resonant interactions between the Stark separation and the oscillations of the dynamic field occur, depressions or "dips" appear on the spectrum. These complex phenomena are described by several authors $[39,40,41,42,43,44,45,46,47]$. The addition of these effects, typical in non-ideal 
plasma, results in multiple modifications in the $\mathrm{H}_{\alpha}$ profile as seen on figure 12 . The complexity of the emission spectrum reflects the complexity of the plasma itself: it is a cold and dense plasma, highly collisional, with strong electric field in the near electrode layers, turbulence and resonance phenomena.

\section{CONCLUSIONS}

Optical emission spectroscopy applied to EDM sparks gives useful informations about the plasma. The electrical discharge cracks almost completely the dielectric molecules. Discharges in oil and water produce strong $\mathrm{H}_{\alpha}$ radiation, which can be used for density measurements. Workpiece erosion or electrode wear regime can also qualitatively be differentiated with spectroscopy. Changing the electrode polarity indeed produces very different spectra. Furthermore, the electrode materials contaminate the plasma: many atomic lines of these materials are clearly visible in spectra. Assuming local thermal equilibrium and applying the Boltzmann plot method with copper lines, the electron temperature is evaluated around $0.7 \mathrm{eV}$. Time-resolved spectra, along with time-integrated spectra of discharges with different on-times, show that the plasma density is above $2 \cdot 10^{18} \mathrm{~cm}^{-3}$ at the beginning of the discharge, and then decreases with time. This very high density produces strong Stark broadening and shift of the $\mathrm{H}_{\alpha}$ line, absence of $\mathrm{H}_{\beta}$ line and merging of all lines in the first $\mu \mathrm{s}$. The low temperature and the high density of EDM plasma make them non-ideal. The plasma coupling parameter can be evaluated and is around 0.45 . The plasma non-ideality has other spectroscopic consequences, such as asymmetry of the $\mathrm{H}_{\alpha}$ line and complex structures around it. EDM discharges produce plasma with extreme properties, which have many interesting spectroscopic consequences. This work gives a first insight into the complexity of EDM plasma, but further investigations remain to be done.

\section{Acknowledgments}

This work is funded by the Swiss Federal Research grant TopNano 21, project $\mathrm{n}^{\circ} 5768.2$.

\section{References}

[1] Germer L H and Haworth F E 1949 J. Appl. Phys. 201085

[2] Cobine J D and Burger E E 1955 J. Appl. Phys. 26895

[3] Zingerman A S 1956 Sov. Phys. Tech. Phys 11945

[4] Kobayashi K 1995 Proc. of the 11th Int. Symposium for Electro Machining (ISEM-11) 29

[5] Loettgen R 1998 Proc. of the 12th Int. Symposium for Electro Machining (ISEM-12) 341

[6] Kruth J P, Toenshoff H K and Klocke F 1998 Proc. of the 12th Int. Symposium for Electro Machining (ISEM-12) 331

[7] Das S, Klotz M and Klocke F 2003 J. Mater. Process. Technol. 142434

[8] Van Dijck F 1973 Physico-mathematical analysis of the electro discharge machining process (Ph.D. dissertation, Catholic University of Leuven, Belgium)

[9] DiBitonto D D, Eubank P T, Patel M R and Barrufet M A 1989 J. Appl. Phys. 664095 
[10] Patel M R, Barrufet M A, Eubank P T and DiBitonto D D 1989 J. Appl. Phys. 664104

[11] Eubank P T, Patel M R, Barrufet M A and Bozkurt B 1993 J. Appl. Phys. 737900

[12] Albinski K, Musiol K, Miernikiewicz A, Labuz S and Malota M 1996 Plasma Sources Sci. Technol. 5736

[13] Pillans B W, Evensen M H, Taylor H F and Eubank P T 2002 J. Appl. Phys. 911780

[14] Devins J, Rzad S and Schwabe R 1981 J. Appl. Phys. 524531

[15] Frayssines P, Bonifaci N, Denat A and Lesaint O 2002 J. Phys. D: Appl. Phys. 35369

[16] Forster E O 1990 J. Phys. D: Appl. Phys. 231506

[17] Robinson J, Ham M and Balaster A 1973 J. Appl. Phys. 4472

[18] Barmann P, Kroell S and Sunesson A 1996 J. Phys. D: Appl. Phys. 291188

[19] Kukhlevsky S V, Kaiser J, Samek O, Liska M and Erostyak J 2000 J. Phys. D: Appl. Phys. 33 1090

[20] Fortov V E and Iakubov I T 1990 Physics of Nonideal Plasma (New York: Hemisphere publishing corp.)

[21] Redmer R 1997 Phys. Rep. 28235

[22] Griem H R 1974 Spectral Line Broadening by Plasmas (New York: Academic Press)

[23] Griem H R 1997 Principles of Plasma Spectroscopy (Cambridge: University Press)

[24] Heading D J, Bennett G R, Wark J S and Lee R W 1995 Phys. Rev. Lett. 743616

[25] Vitel Y, El Bezzari M, Gavrilova T V, Skowronek M, D'yachkov L G and Kurilenkov Y K 1996 Physica B 228161

[26] Escarguel A, Ferhat B, Lesage A and Richou J 2000 J. Quant. Spectrosc. Radiat. Transfer 64353

[27] Gigosos M A and Cardenoso V 1996 J. Phys. B: At. Mol. Opt. Phys. 294795

[28] Boeddeker S, Guenter S, Koenies A, Hitzschke L and Kunze H-J 1993 Phys. Rev. E 472785

[29] Olchawa W 2002 J. Quant. Spectrosc. Radiat. Transfer $\mathbf{7 4} 417$

[30] Flih S A, Oks E and Vitel Y 2003 J. Phys. B: At. Mol. Opt. Phys. 36283

[31] Guenter S and Koenies A 1997 Phys. Rev. E 55907

[32] Escarguel A, Oks E, Richou J and Volodko D 2000 Phys. Rev. E 622667

[33] Lesaint O and Gournay P 1994 IEEE Trans. on Diel. and Electr. Insul. 1702

[34] Beroual A, Zahn M, Badent A, Kist K, Schwabe A J, Yamashita H, Yamazawa K, Danikas M, Chadband W G and Torshin Y 1998 IEEE Electr. Insul. Mag. 146

[35] Inglis D R and Teller E 1939 Ap. J. 90439

[36] Vidal C-R 1966 J. Quant. Spectrosc. Radiat. Transfer 6461

[37] Roussel K M and O'Connell R F 1975 Phys. Lett. 51244

[38] Kuhn H G 1962 Atomic Spectra (London: Longmans)

[39] Cohn A, Bakshi P and Kalman G 1972 Phys. Rev. Lett. 29324

[40] Gallagher C C and Levine M A 1973 Phys. Rev. Lett. 30897

[41] Rutgers W R and Kalfsbeek H W 1975 Z. Naturforsch. 30739

[42] Zhuzhunashvili A I and Oks E A 1977 Sov. Phys. JETP 461122

[43] Finken K H, Buchwald R, Bertschinger G and Kunze H-J 1980 Phys. Rev. A 21200

[44] Gavrilenko V P and Oks E A 1983 Sov. J. Plasma Phys. 1322

[45] Kalman G and Bakshi P 1990 J. Quant. Spectrosc. Radiat. Transfer 441

[46] Bakshi P and Kalman G 1990 J. Quant. Spectrosc. Radiat. Transfer 4493

[47] Oks E A, Boedekker S and Kunze H-J 1991 Phys. Rev. A 448338 